\title{
BMJ Open Real-world treatment persistence of golimumab in the management of immune-mediated rheumatic diseases in Europe: a systematic literature review
}

\author{
Karin Luttropp, ${ }^{\oplus 1}$ Mary Dozier, ${ }^{2}$ Nahila Justo, ${ }^{1,3}$ Freddy Cornillie, ${ }^{4}$ \\ Sumesh Kachroo, ${ }^{5}$ Marinella Govoni, ${ }^{6}$ Stina Salomonsson, ${ }^{7}$ Christopher M Black, ${ }^{5}$ \\ Ahmed Khalifa ${ }^{4}$
}

To cite: Luttropp K, Dozier M, Justo $\mathrm{N}$, et al. Real-world treatment persistence of golimumab in the management of immune-mediated rheumatic diseases in Europe: a systematic literature review. BMJ Open 2019;0:e27456. doi:10.1136/ bmjopen-2018-027456

- Prepublication history and additional material for this paper are available online. To view these files, please visit the journal online (http://dx.doi org/10.1136/bmjopen-2018027456).

Received 23 October 2018 Revised 14 February 2019 Accepted 25 March 2019
Check for updates

(C) Author(s) (or their employer(s)) 2019. Re-use permitted under CC BY-NC. No commercial re-use. See rights and permissions. Published by BMJ.

For numbered affiliations see end of article.

Correspondence to

Dr Karin Luttropp;

karin.luttropp@iconplc.com

\section{ABSTRACT}

Objectives To summarise real-world data from studies reporting golimumab persistence in European immunemediated rheumatic disease (IMRD) populations and to report pooled estimates.

Design Systematic literature review.

Data sources Relevant literature was identified through searching Medline and Embase via Ovid as well as the conference databases of European League Against Rheumatism and American College of Rheumatology Association of Rheumatology Health Professionals. Eligibility criteria We screened records using predefined patients, interventions, comparators, outcomes and study design criteria. Eligible studies included reports of persistence among adult IMRD patients in Europe receiving treatment with subcutaneous golimumab. Clinical trials, randomised controlled trials, literature reviews, editorials, guidelines and studies with $<20$ patients receiving golimumab were excluded.

Data extraction and synthesis Following double screening by two independent reviewers, 27 studies out of 578 identified records were selected for inclusion and subsequent data extraction. Persistence was most commonly reported at 12 and 24 months; hence, pooled persistence estimates were calculated for these two time points and reported according to indication.

Results Persistence ranged between $58.1 \%$ (psoriatic arthritis (PsA) patients regardless of treatment line) and $75.7 \%$ (biological-naïve rheumatoid arthritis patients) at 12 months; at 24 months, the range was $43 \%$ (axial spondyloarthritis $(\mathrm{AxSpA})$ patients regardless of treatment line) and $69.6 \%$ (biological-naïve PsA patients). On the basis of data from 12 studies, persistence with golimumab treatment was either significantly higher or not significantly different from other tumour necrosis factor inhibitors (TNFi).

Conclusions Golimumab persistence at 24 months approximates $50 \%$, with a lower persistence among AxSpA (43\%) patients. However, as the number of studies in these populations was low, they warrant further research. In 12 studies comparing various TNFi treatments, golimumab was shown to have significantly better or equal persistence to its comparators.
Strengths and limitations of this study

- By excluding data from randomised controlled trials, this systematic literature review provides an up-todate overview of real-world tumour necrosis factor inhibitors persistence in European immune-mediated rheumatic disease (IMRD) patient populations.

- Including different IMRD diagnoses, as opposed to limiting the scope to a single rheumatic disease, allows comparisons of treatment patterns across various disorders.

- The definition of persistence varied across included studies, thereby complicating the interpretation of any direct comparisons of results.

\section{INTRODUCTION}

The collective term immune-mediated rheumatic diseases (IMRD) encompasses a group of diagnoses, including axial spondyloarthritis (AxSpA), psoriatic arthritis (PsA) and rheumatoid arthritis (RA). Common to all types of IMRD is the presence of chronic inflammation, as evidenced by elevated synovial joint fluid levels of tumour necrosis factor alpha (TNF- $\alpha)$, which, in turn, stimulates the production of inflammatory cytokines, such as interleukin (IL)-1, IL-6, granulocyte macrophage colony stimulating factor (CSF) and granulocyte CSF. ${ }^{12}$ The landscape for treatment of IMRD has evolved rapidly, and currently includes several TNF- $\alpha$ inhibitors (TNFi) in addition to the conventional synthetic disease-modifying antirheumatic drugs (DMARDs) and non-steroidal anti-inflammatory drugs (NSAIDs) commonly used to treat IMRD. Other treatments, targeting IL-17 and Janus kinase pathways as well as the lymphocytes involved in propagating the inflammatory response, are also approved for clinical use or are still investigational. ${ }^{3-5}$ 
In Europe, the subcutaneously administered TNFi golimumab is indicated for the treatment of active RA, AxSpA and PsA. ${ }^{6}$ Following the randomised controlled trials GO-FORWARD (RA patients), ${ }^{7}$ GO-RAISE (ankylosing spondylitis (AS) patients), ${ }^{8}$ GO-REVEAL (PsA patients) ${ }^{9}$ and GO-AHEAD (non-radiographic AxSpA patients), ${ }^{10}$ which established the efficacy and safety of golimumab, several open-label extension (OLE) studies were conducted. ${ }^{11-13}$ The OLE studies followed patients for 5 years to obtain long-term outcome data, including persistence, defined as the proportion of patients not discontinuing treatment. OLE studies of golimumab in biological-naïve IMRD patients showed a 5-year persistence rate of $70 \%$; among biological-experienced RA patients, the corresponding rate was approximately $40 \% .^{11-15}$ However, patients enrolled in randomised controlled trials and subsequently, OLE studies are not always representative of patients in clinical practice. Thus, real-world data on drug utilisation, treatment patterns and outcomes are of great importance, as they provide information regarding the usage of available treatments outside of the strictly controlled environment of randomised controlled trials. Real-world persistence with treatment is particularly useful, as it has been considered a proxy marker not only for treatment safety and effectiveness, but also for patient satisfaction. ${ }^{16-18}$ The definition of persistence is the 'duration of time from initiation to discontinuation of therapy', while two other commonly used terms, adherence and compliance, are related to patient behaviour and conformance with the prescribed dosing regimen. ${ }^{19}$ Several systematic literature reviews (SLRs) focusing on real-world treatment patterns of golimumab and other TNFi in IMRD populations have been published. ${ }^{20-24}$ However, many focus on a particular subset of IMRD patients-such as RA populations-and do not include patients with other IMRD indications that also receive TNFi treatment; in addition, recent years have seen a substantial increase in studies reporting realworld evidence of TNFi treatment patterns, warranting an updated overview of the field.

\section{METHODS}

In accordance with the study protocol (available on request), the literature search was performed in Medline and Embase via Ovid as well as in the American College of Rheumatology-Association of Rheumatology Health Professionals (ACR-ARHP) and European League Against Rheumatism (EULAR) conference abstract databases. The search was limited to include all studies indexed in Medline or Embase from 01 January 2009 to 30 April 2018. For EULAR abstracts, the search was limited to conference papers from 2017 to 2018, and for ACR-ARHP, only abstracts from the 2017 conference were considered. The search strings applied are listed in online supplementary tables 1-3. As golimumab is available as an intravenously administered formulation in non-European regions, with a label and dosage differing from the label approved by the European Medicines Agency (EMA), the literature search was limited to European studies including only the subcutaneous formulation and dosage approved by EMA. ${ }^{6}$ The search was restricted to studies published in English.

The identified publications were subsequently entered into an abstract screening sheet and duplicates were removed. Where several publications reported the results for the same patient population, only one was included.

This study was conducted in accordance with the Preferred Reporting Items for Systematic Reviews and Meta-Analyses (PRISMA) guidelines. ${ }^{25}$ Hence, all studies identified in the original literature search were assessed for inclusion eligibility using the patients, interventions, comparators, outcomes and study design (PICOS) approach, as outlined below:

\section{Patients}

Studies including adult patients ( $\geq 18$ years of age $)$ in Europe diagnosed with IMRD, including AxSpA (encompassing AS and non-radiographic AxSpA), PsA and RA, were eligible for inclusion. Studies reporting joint results for mixed IMRD populations were also included but are presented separately.

\section{Interventions}

Studies reporting data on golimumab, administered subcutaneously, were eligible for inclusion. Studies where the mode of administration was reported as intravenous, were excluded.

\section{Comparators}

No restrictions applied.

\section{Outcomes}

Studies reporting estimates of persistence were eligible for inclusion. This included data on the proportion of patients persistent with golimumab treatment at specific time points (persistence rates at different landmarks); mean or median time to golimumab treatment discontinuation (time to event); and/or mean or median time until $50 \%$ of the patient population are no longer persistent with golimumab treatment (treatment survival).

\section{Study design}

All studies reporting real-world evidence, prospective or retrospective, were eligible for inclusion. Pivotal registration clinical trials, literature reviews, editorials, guidelines and studies with $<20$ patients receiving golimumab were excluded.

Two independent reviewers using the PICOS criteria outlined above screened abstracts of the identified studies, and individual decisions on whether to include or exclude individual studies in the review were aligned until a consensus decision was reached. A second screening step was made based on the full-text version of the studies (where available), using the same approach as the one utilised in the abstract screening stage. 


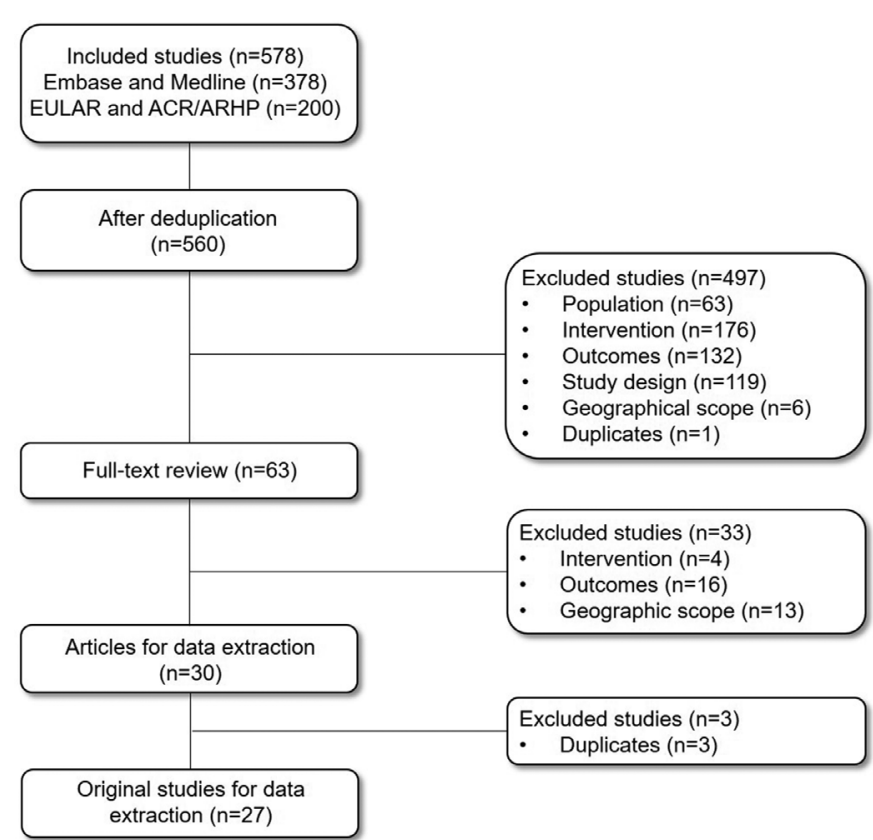

Figure 1 Selection process flowchart for identification of studies to be included in the literature review. ACR, American College of Rheumatology; ARHP, Association of Rheumatology Health Professionals; EULAR, European League Against Rheumatism.

Following the completion of the full-text screening, data were extracted from the studies included in the review. Information was extracted regarding (1) reference details; (2) study design; (3) patients; (4) treatments; (5) outcomes and (6) geographic scope. A full list of extracted variables is available in online supplementary table 4 . Results were reported as pooled estimates of persistence by indication, weighted according to the number of patients in each subpopulation. For each subgroup analysis, it was noted which of the included studies had undertaken measures to minimise bias, and which had not. Studies that did not report the number of patients were not included in the pooled persistence estimates.

\section{Risk of bias assessment}

Studies included in the review were subjected to a risk of bias assessment using the Newcastle-Ottawa scale for cohort studies. $^{26}$ The scale was modified slightly so that the maximum score for selection was two points, since the categories 'selection of the non-exposed cohort' and 'demonstration that outcome of interest was not present at the start of the study' were set as 'not applicable' for all studies included in the current review. The results of the assessment are reported in online supplementary file S1. A meta-analysis was not deemed feasible based on the studies selected for inclusion, and hence, no assessment of systematic bias across studies was performed.

\section{Patient and public involvement}

Since the current study is an SLR, there was no involvement of patients and/or the public.
RESULTS

Identification and inclusion of studies

The Ovid search generated 378 hits, while the searches of the ACR-ARHP and EULAR database searches generated 16 and 184 hits, respectively. Duplicates were identified and removed, resulting in 560 full-text articles and conference abstracts that were included in the initial abstract screening. Sixty-three of these were included in a full-text screening, of which 30 records were selected for data extraction. Three studies were reported as both abstracts and full-texts; thus, only the estimates reported in the full-texts were included in the pooled results, resulting in 27 included studies. The selection process is shown in figure 1, using a PRISMA flowchart.

\section{Study and patient characteristics}

Of the studies included in the literature review, 9 were fulltext articles ${ }^{27-35}$ while 18 were conference abstracts. ${ }^{36-53}$ The years of data collection ranged from June $2003^{43}$ to March 2016. ${ }^{42}$ Seven studies did not report the period for data collection ${ }^{45-4850-52}$; five of these were prospective studies. ${ }^{47450-52}$ The maximum number of months during which golimumab treatment persistence was reported ranged from $6^{50}$ to 60 months, ${ }^{43} 45$ the most common time points for reporting outcomes being 12 months (reported in 16 studies) $^{27-3134-4042444547}$ and 24 months (reported in 13 studies). ${ }^{27-33} 35 \quad 3641 \quad 44-46$ Ten studies reported golimumab persistence at more than one time point. ${ }^{27-313536444547}$ A minority (5/27) of the included studies reported data on mean/median persistence in months. ${ }^{32} 35495152$ Four studies reported using methods to address selection bias, ${ }^{29-3153}$ while an additional five studies used Cox proportional hazards model to identify potential confounders. ${ }^{27} 28333452$ The remaining 18 studies did not specify which methods, if any, that were used to address bias $^{32}{ }^{35-51} ; 16$ of these were conference abstracts. $^{36-51}$

Of the 19 studies that reported gender distribution in the patient cohort, the proportion of females ranged from $38.8 \%$ (AS patient subpopulation) ${ }^{51}$ to $89.2 \%$ (RA patient subpopulation). ${ }^{35}$ Eight studies did not report the proportion of female versus male patients ${ }^{36-40464749}$; these were all conference abstracts. The same eight studies did not report mean or median age of patients included in the cohorts. The reported mean age ranged between $39.8 \pm 10.1$ years (nr-AxSpA patient subpopulation $)^{51}$ and $58.3 \pm 13.3$ years (RA patient subpopulation). ${ }^{35}$ Two studies reported median as opposed to mean age; one investigating PsA patients (median age 50 years (IQR $41-57)^{27}$ ) and one studying RA patients (median age 55 years (IQR 46-62) ${ }^{28}$ ).

One study reported exclusively on an AS population ${ }^{38}$; five reported exclusively on a PsA population 2739435052 and six studies reported exclusively on an RA population. ${ }^{283134404146}$ Fourteen studies ${ }^{29} 303233$ 35-37 $42444547-4952$ included at least two different IMRD patient populations, and one study ${ }^{53}$ did not provide any data on disease distribution within the cohort. Mean or median disease 
duration was reported in 13 studies, 2728 31-33 $354144-46485152$ and ranged between a median of 6.1 years (IQR 1.7-13) among a cohort of PsA patients ${ }^{27}$ and a mean of 18.8 years (SD 10.1) in a cohort of AS patients. ${ }^{51}$ A total of 19 studies reported patient treatment history with regard to previous usage of biologic treatments; the proportion of biological-naïve patients ranged from 30\% (AS patient subpopulation) ${ }^{35}$ to $63 \%$ (IMRD patient population). ${ }^{42}$ Four 30343743 and three studies ${ }^{29} 3144$ reported exclusively on naïve and experienced patient populations, respectively.

Table 1 provides an overview of selected patient characteristics of the cohorts included in the literature review; it also includes the concept of persistence used in each study, defined as the time period during which patients are required to fill their prescriptions before a specified number of days elapses (sometimes referred to as the 'gap size').

Pooled golimumab persistence estimates at 12 and 24 months Figure 2 illustrates the proportion of patients persistent with golimumab treatment at specific time points by indication. The reporting time points ranged between 3 months ${ }^{27-29} 47$ and 60 months, ${ }^{43} 45$ the latter of which is comparable to the outcome reporting time point used in OLEs of the golimumab randomised controlled trials. As can be seen from figure 2 , the most commonly reported time points were 12 and 24 months. At 12 months, the total number of studies reporting golimumab persistence ranged from five for AxSpA patients ${ }^{35-38} 44$ to eight for IMRD patients 2930353642444547 ; at 24 months, the number of studies ranged between three and eight for $\mathrm{AxSpA}^{35} 3644$ and RA, ${ }^{28} 31-3335364146$ respectively. A detailed overview of the reporting time points is provided in online supplementary table 5 . Pooled golimumab persistence data from 12 and 24 months, weighted according to relative sample size, are presented in figure 3A-D. The study by Favalli $e t$ $a l^{41}$ was excluded from further analysis following quality assessment, and thus was not included in the pooled estimates. Note that since a different number of studies was used to calculate each pooled estimate, the number of patients used to weigh the sample size also varied between the pooled estimates.

\section{Mixed IMRD populations}

The persistence resulting from pooling estimates reported from overall IMRD patient populations treated with golimumab regardless of treatment line was $60.4 \%$ at 12 months 2930353642444547 and $58.6 \%$ at 24 months. $^{293032354445}$ For biological-naïve IMRD patients, the pooled persistence at 12 months was $63.8 \%$; at 24 months, it was $52.2 \% .^{30}$ Biological-experienced IMRD patients receiving golimumab in second and subsequent lines had a persistence rate of $71.7 \%$ and $58.7 \%$ at 12 and 24 months, respectively ${ }^{294445}$ (figure 3A).

\section{RA populations}

For RA, patients treated with golimumab had a pooled persistence across all therapy lines of $62.3 \%$ at 12 months $^{28} 3134-36$ and $49.8 \%$ at 24 months $2831-33353646$ (figure 3B). Biological-naïve RA had a pooled 12-month persistence rate of $75.7 \%^{28}$ and $62.5 \%$ at 24 months. $^{28} 3233$ Biological-experienced RA patients had a pooled persistence rate of $71.3 \%$ at 12 months, ${ }^{28} 31$ while at 24 months, the level of pooled persistence was $58.1 \% .^{2831-33}$

\section{AxSpA populations}

When analysing AxSpA populations regardless of treatment line, the pooled persistence was $55.4 \%$ at 12 months ${ }^{3536}$ and $43 \%$ at 24 months ${ }^{33} 3536$ (figure 3C). Only two studies reported persistence by treatment line (one for biological-naïve patients and one for biological-experienced patients); therefore, no pooled persistence was calculated for these.

\section{PsA populations}

Regardless of treatment line, PsA patient populations had a pooled golimumab persistence of $58.1 \%$ at 12 months 27353644 ; at 24 months, the pooled persistence was 48.7\%. 273233353644 While no pooled estimate for biological-naïve PsA patients could be calculated at 12 months since it was only reported in one study, ${ }^{27}$ the persistence rate at 24 months was $69.6 \% .^{2733}$ For biological-experienced PsA patients, golimumab persistence was $74.7 \%$ at 12 months ${ }^{27} 44$ and $63 \%$ at 24 months ${ }^{2734} 44$ (figure 3D).

\section{Persistence expressed as mean number of months}

For mean golimumab persistence expressed as mean number of months until discontinuation, pooled data were only available for PsA and RA populations with mixed or unknown treatment history. For IMRD and AxSpA, as well as for subpopulations with specified treatment history, only one study reported mean survival time. The pooled mean survival time is shown in figure 4 . For PsA, mean survival time in months equalled $26.3( \pm 2.6)$ (three studies) $^{32} 3552$ (figure $4 \mathrm{~A}$ ); for RA, it was determined as 33 months $( \pm 1.8)$ (two studies) ${ }^{3235}$ (figure $4 \mathrm{~B}$ ).

\section{Comparative persistence}

In total, 12 studies reported persistence of golimumab and other biological treatments. ${ }^{27-3137-414353}$ All of these studies included data on adalimumab and etanercept, while nine studies included data on certolizumab pegol ${ }^{28-30} 37-4153$ and three on infliximab. ${ }^{272843}$ Four studies reported effect size of the difference in persistence, formulated as hazard ratios (HRs), using golimumab as the reference treatment. ${ }^{28}{ }^{38-40}$ Of these, golimumab persistence was signifi-

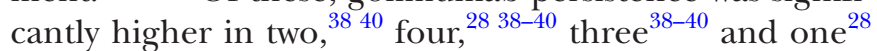
study when compared with adalimumab, certolizumab pegol, etanercept and infliximab, respectively. An overview of studies reporting differences in effect size HR is provided in figure 5 , in which an $\mathrm{HR}>1$ indicates that golimumab persistence is significantly higher than that of its comparator. The results shown in figure 5 were further confirmed in studies reporting significant differences in persistence compared with golimumab as $p$ values only; for adalimumab, ${ }^{29-31} 53$ etanercept $^{30}$ and infliximab, ${ }^{43}$ 


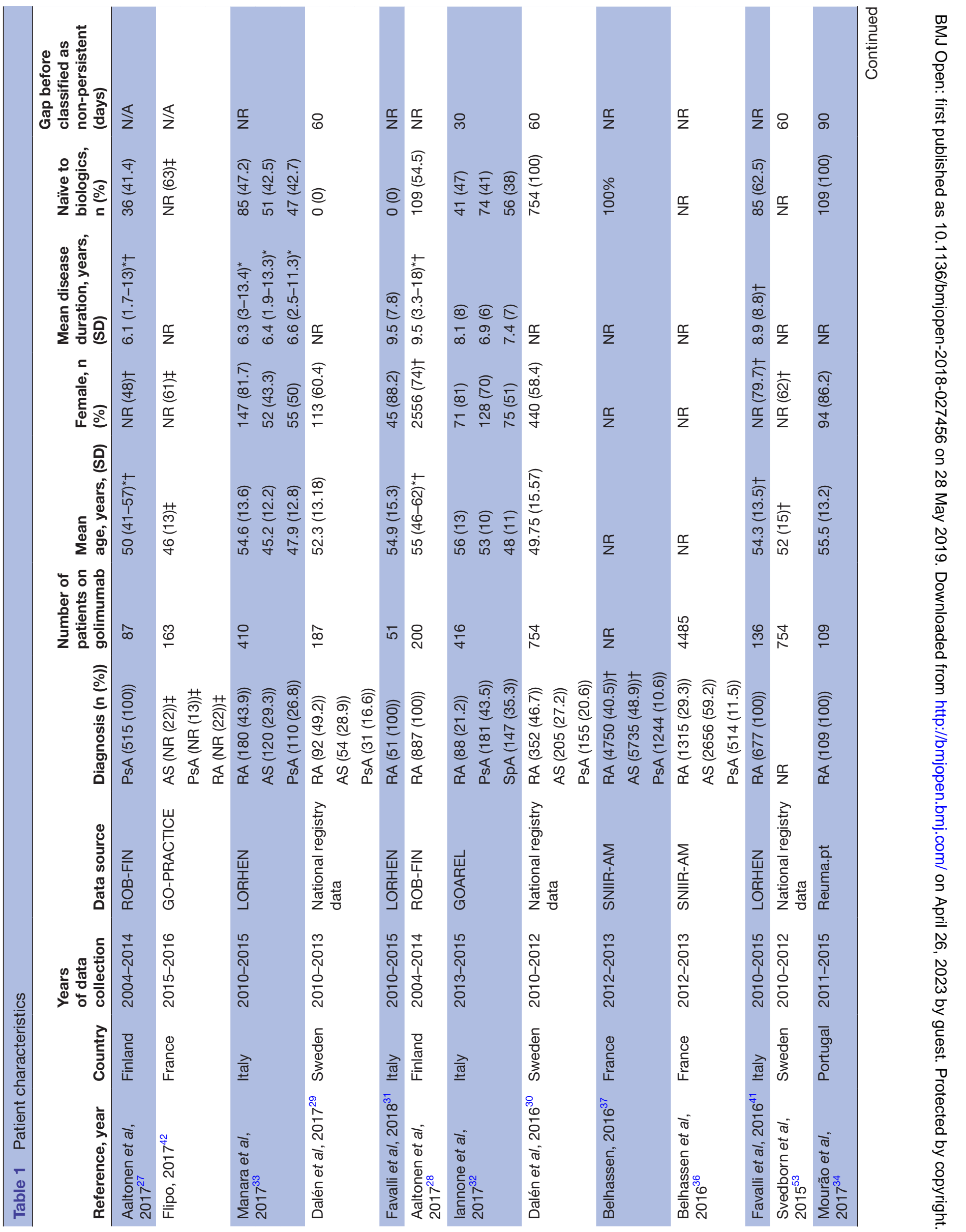




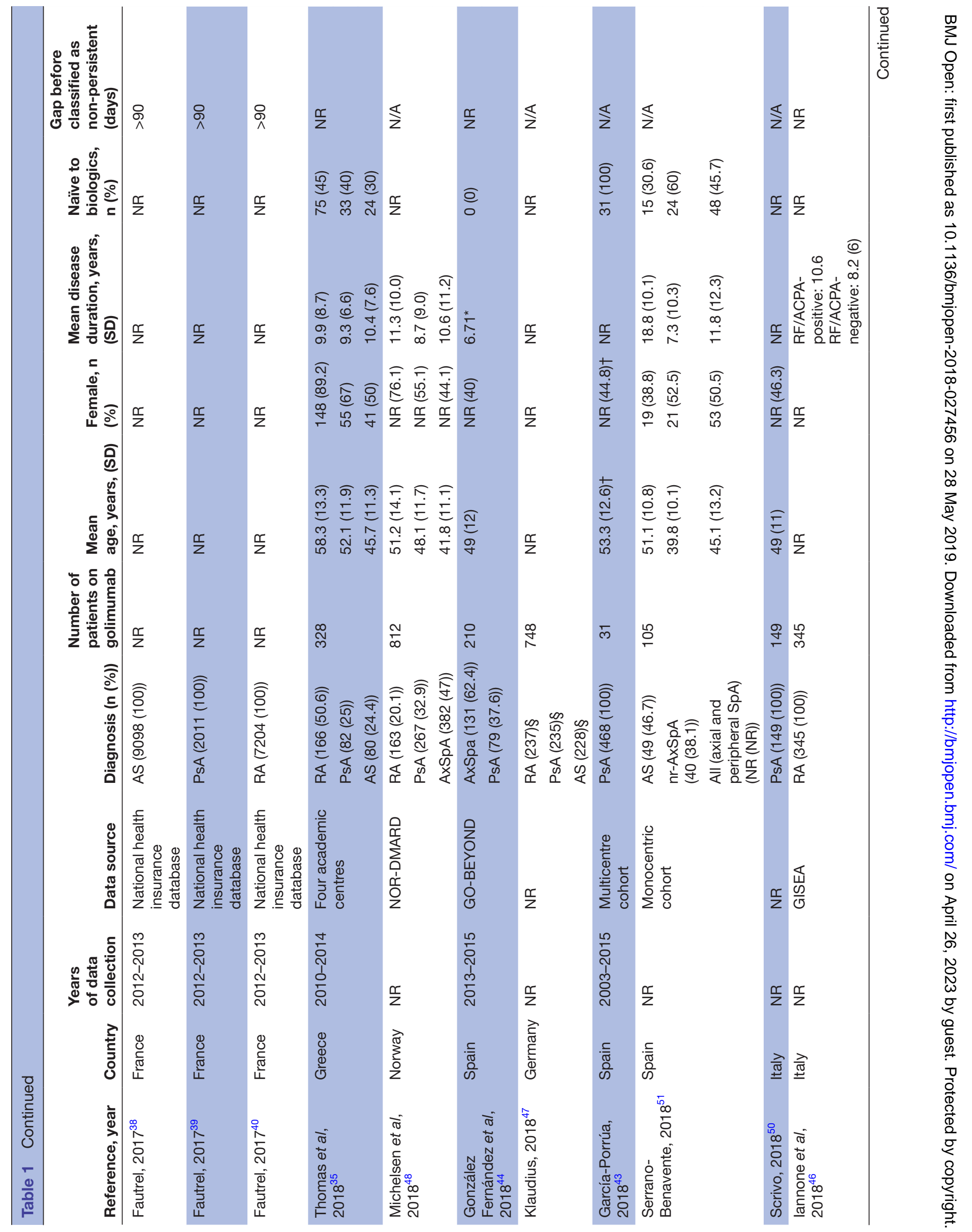




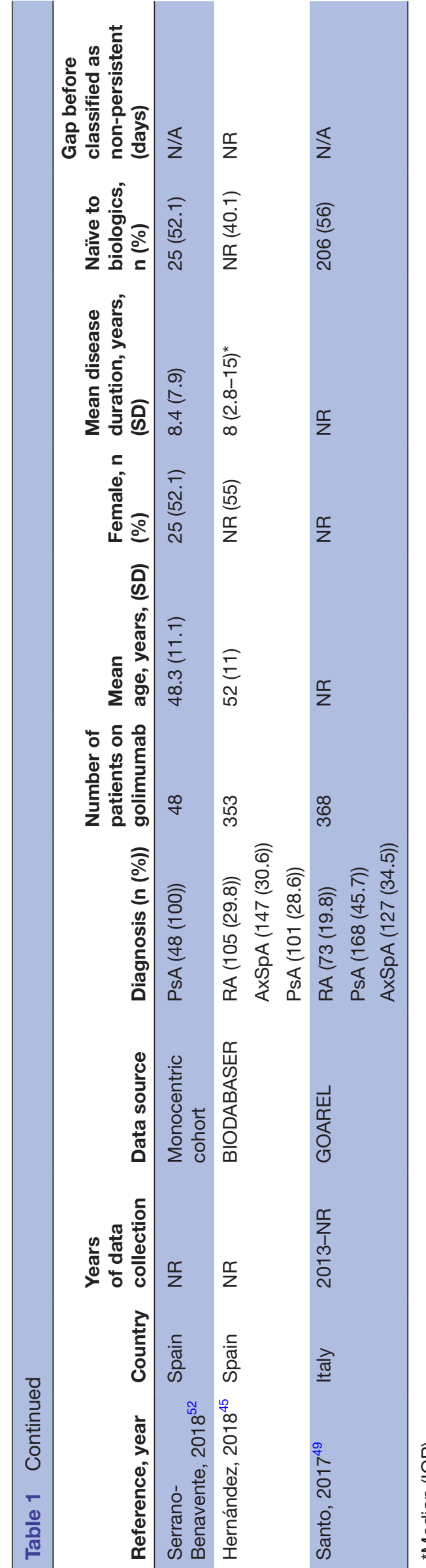

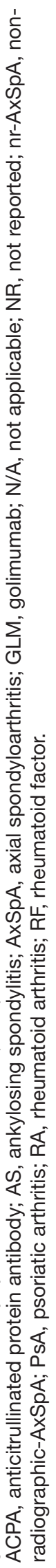

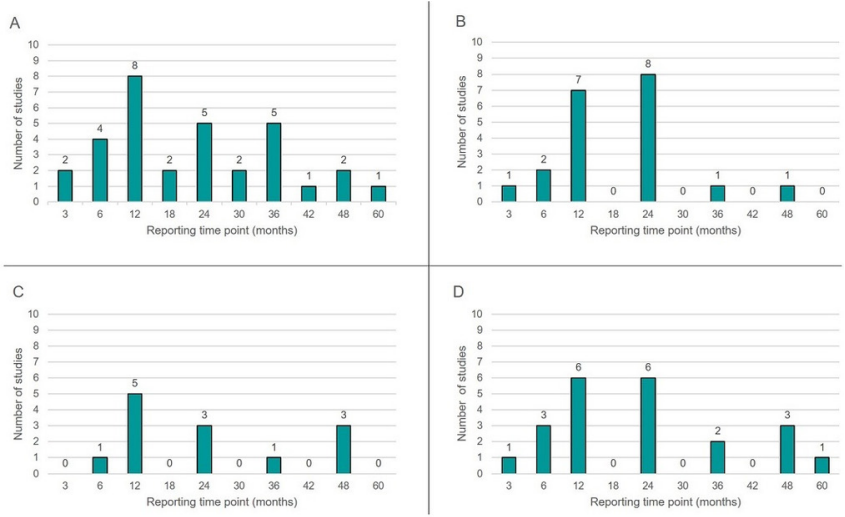

Figure 2 Number of studies reporting persistence at various time points. Results are reported by indication. (A) Immunemediated rheumatic disease; (B) rheumatoid arthritis; (C) axial spondyloarthritis and (D) psoriatic arthritis.

respectively. Remaining comparisons in persistence between golimumab and adalimumab, certolizumab pegol, etanercept or infliximab showed no significant differences. ${ }^{27-31} 394153$ A detailed summary of comparative persistence data can be found in online supplementary table 6 .

Compared with adalimumab, golimumab was reported to have significantly higher persistence in seven studies, ${ }^{29-3138404153}$ while two studies found no significant difference between the two comparators ${ }^{2839}$; three studies reported persistence for adalimumab and golimumab, but did not provide any $p$ values or results from statistical tests between them. ${ }^{27}{ }^{37} 43$ Compared with certolizumab pegol, four studies found no significant difference in persistence, ${ }^{29} 304153$ while four other studies found that golimumab had a significantly higher persistence ${ }^{28} 38-40$; one study did not provide any information on statistical significance between patients receiving certolizumab pegol or golimumab. ${ }^{37}$ Compared with etanercept,

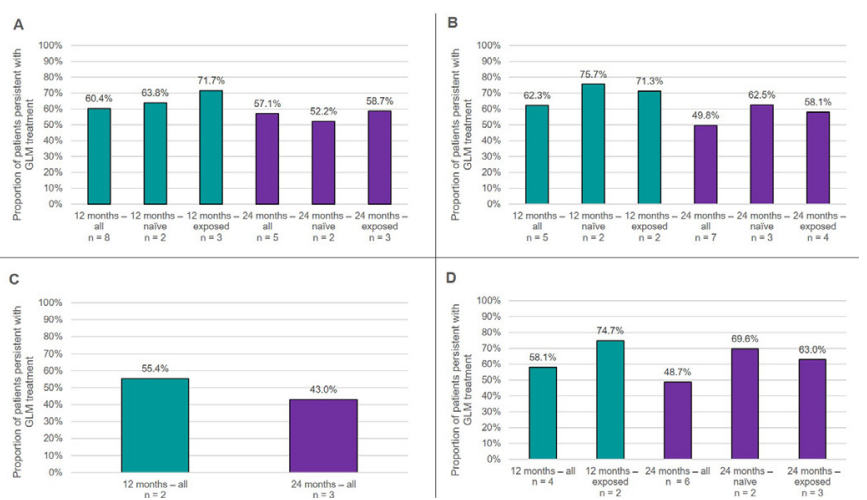

Figure 3 Pooled persistence data at 12 and 24 months, by treatment line (where available). Results are reported by disease. (A) IMRD populations (persistence data not reported for individual diagnoses) 2930353642444547 ; (B) rheumatoid arthritis populations s $^{31-3646}$; (C) axial spondyloarthritis populations ${ }^{333536}$ and (D) psoriatic arthritis populations. 273233353644 IMRD, immune-mediated rheumatic disease; GLM, golimumab; n, number of studies included. 

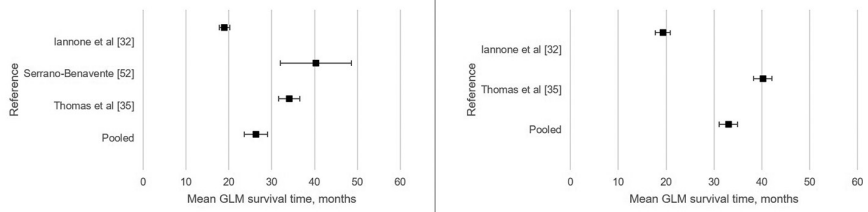

Figure 4 Pooled mean survival time (months) for patients with mixed or unknown treatment history receiving golimumab (GLM) treatment. (A) Psoriatic arthritis patient populations ${ }^{32} 3552$ and $(\mathrm{B})$ rheumatoid arthritis patient populations. $^{32} 35$

golimumab was found to have higher persistence in five studies $^{30} 38-4053$; four studies could not detect any significant difference between the two comparators 28293141 and three studies did not provide any information on statistical significance between patients receiving etanercept or golimumab. ${ }^{27} 3743$ Compared with infliximab, golimumab was found to have significantly higher persistence in one study ${ }^{28}$; no significant difference in persistence in another ${ }^{27}$ and numerically higher persistence in a third study, which did not report any information on statistical significance. ${ }^{43}$ Overall, golimumab shows statistically higher persistence or at least numerically higher persistence when compared with other TNFi treatments based on reported HR and p-values.

\section{Predictors of persistence}

Thirteen studies reported on the predictors of persistence among patients taking golimumab. ${ }^{32} 343538-4043454648495152$ Eight studies found females to be significantly less persistent. ${ }^{32} 3438-40434849$ Biological-experienced patients were significantly less persistent in six studies ${ }^{38-40} 454652$ while four studies found no difference between biological-naïve and biological-experienced patients. 34354651 'No concomitant DMARDs' were found to be a predictor of low persistence in three

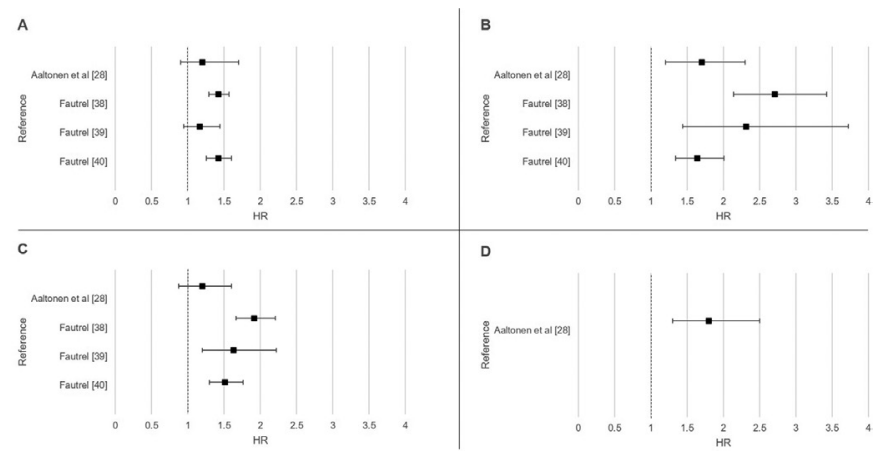

Figure 5 Effect size of differences in persistence between golimumab (GLM) and comparators, expressed as HRs. All $\mathrm{HR}$ calculations were made using GLM persistence data as reference. (A) GLM versus adalimumab; (B) GLM versus certolizumab pegol; (C) GLM versus etanercept and (D) GLM versus infliximab. $H R>1$ indicates that comparator persistence is lower than reference (GLM) treatment persistence. studies. $^{3243} 48$ A summary of the reported predictors of persistence across studies is presented in table 2 .

\section{DISCUSSION}

This study provides an up-to-date overview of persistence with golimumab among IMRD patients in Europe. By excluding randomised controlled trial data, the included studies provide valuable information on the real-world persistence of golimumab, as well as information on comparative persistence between golimumab and other TNFi treatments. Hence, the current study offers opportunities for understanding the treatment landscape and improving disease management among IMRD patients.

From a clinical perspective, a high level of persistence with treatment is desirable as it may serve as a proxy for treatment efficacy, safety and patient satisfaction. ${ }^{16-18}$ Furthermore, increased persistence is of interest from a payer perspective, as non-persistent RA patients have been found to incur higher healthcare costs 12 months after switching treatment, compared with patients who are persistent with their index treatment. ${ }^{54}$

The pooled persistence at 12 months ranged between $58.1 \%$ (PsA patients regardless of treatment history) and $75.7 \%$ (biological-naïve RA patients); at 24 months, persistence ranged between $43 \%$ (AS patients regardless of treatment history) and 69.6\% (biological-naïve PsA patients).

Several studies found that the persistence rate of golimumab was higher than that of adalimumab, certolizumab pegol, etanercept and/or infliximab, and no study found golimumab to have a significantly lower persistence than its comparators. Taken together, this overview suggests that golimumab offers a good balance of efficacy, safety and patient satisfaction regardless of current treatment line.

When looking at differences in persistence by indication, golimumab persistence appears to be somewhat higher among RA and PsA patients compared with AxSpA patients; however, as the number of studies is low (particularly for AxSpA), comparisons should be interpreted with caution. In fact, several studies found no differences in persistence between patients with different indications. $^{354548}$ Similarly, although the current SLR did not allow for any statistical comparisons to be made, the data appear to indicate that golimumab persistence does not differ substantially between biological-naïve and biological-experienced IMRD patients.

The most common predictor of non-persistence was being female, resulting in a risk of lower persistence. While being biological-experienced was the second most reported predictor of non-persistence, several studies found no difference in the risk of non-persistence when comparing biological-naïve and biological-experienced, a finding that may further support comparable golimumab persistence in IMRD patients with or without previous exposure to a biological anti-TNF agent. 


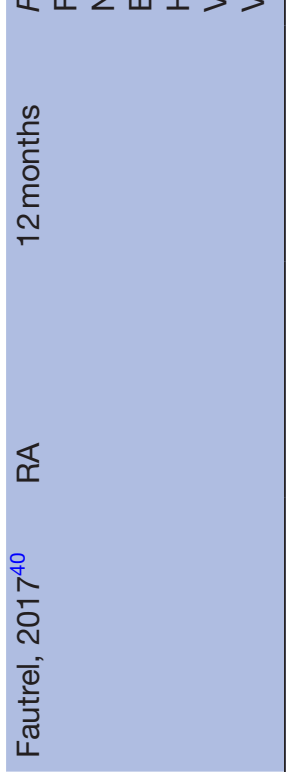

ำ

$\stackrel{n}{\stackrel{n}{*}}$

$\llbracket$

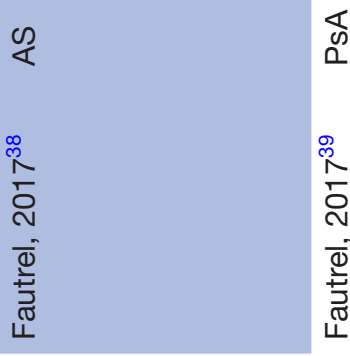

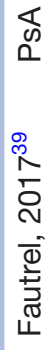




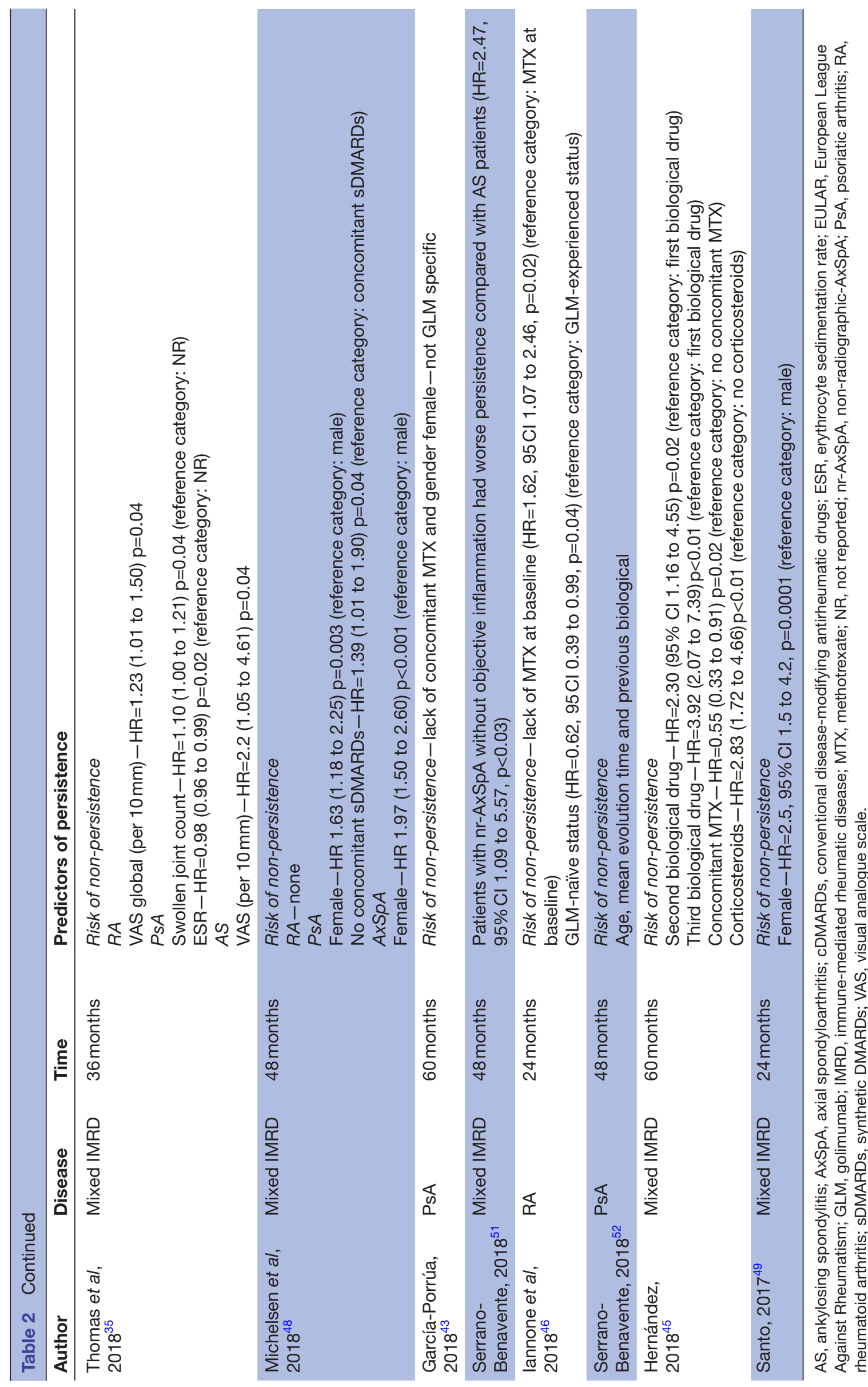

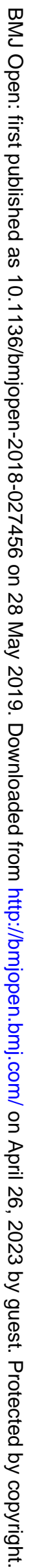


The current study has a few limitations. First, the search string did not include the golimumab brand name Simponi, which may have affected the number of studies identified as part of the Ovid and conference database search. Also, while eligible abstracts from choice conferences were included, other grey literature was not searched since the focus of the current review was directed towards peer-reviewed literature and findings reported in a conference setting. Second, studies included in this SLR did not allow for a meta-analysis to be performed, as there were too few studies reporting persistence by indication for any particular time point. Because no meta-analysis was performed, no formal assessment of selection bias was done; however, study quality was assessed using the Newcastle-Ottawa scale for cohort studies, ${ }^{26}$ the results of which are provided in online supplementary file S1. Overall, study quality did not vary significantly across included studies. Compared with RA and PsA patient populations, the paucity of studies was particularly pronounced for AxSpA, highlighting the need for further research. As several of the included studies were conference abstracts, the amount of available information was, at times, limited. Notably, both Belhassen $e t a l^{36}$ and Fautrel $^{39}$ report the same persistence rate at 12 months among PsA patients (50.8\%); however, since it could not be determined whether the same population was used, both studies were included in the current review. The sparsity of information regarding the definition of persistence used in the included studies is also worth noting. A minority of studies $(8 / 27)$ provide information on the number of days allowed to elapse without re-filling a previously finished prescription before a patient is considered non-persistent; however, the majority (19/27) do not state what gap size was allowed before patients were deemed to have discontinued treatment. Furthermore, known gap size ranged from 30 days $^{32}$ to 91 days, ${ }^{38-40}$ complicating the interpretation of interstudy persistence comparisons.

In addition, since no statistical comparison of baseline characteristics was made between populations included in the pooled persistence estimate, the estimates are potentially exposed to bias provided that baseline characteristics, such as gender, age, comorbidity profile, disease activity and duration, and so on, affect persistence. In addition, since pooled persistence estimates were calculated based on relative sample size, studies not reporting the number of golimumab patients were not included in the weighted average. Similarly, pooled estimates were only calculated for studies that reported persistence at 12 and 24 months.

Finally, patient populations eligible for inclusion were further restricted to studies performed in Europe; while this increases the likelihood that drug administration and dosing is homogenous and in accordance with EMA-approved drug use, it is not possible to extrapolate the findings to non-European patient populations. Also, there are local variations in healthcare systems across Europe with regard to out-of-pocket payments, and so on, which may have had an impact on treatment persistence. Indeed, Giacomelli et al show differences in the use of golimumab when comparing Italian RA patients to patients from other countries. ${ }^{55}$ Similarly, the language restriction (English only) confers an element of publication bias.

The study also has a number of strengths. By restricting the included studies to those reporting real-world data, as opposed to findings made in randomised controlled trials, this review offers an overview of persistence data obtained from actual clinical practice. In a real-world setting, a number of factors interact to determine treatment persistence, for example, adverse effects, loss of efficacy and patient preference. By using persistence as the outcome of interest, this review captures all of the aforementioned factors, regardless of the underlying reason behind treatment switch or cessation. The concept of persistence is, therefore, a simple and useful way of investigating various reasons for treatment switch and discontinuation simultaneously. Furthermore, by grouping persistence data by indication and by investigating differences in persistence between different types of TNFi, the current review highlights differences in persistence between various patient groups and TNFi treatments. Finally, this study adds to previous studies of similar design by expanding the number of included studies, and thereby the available data, detailing realworld persistence among IMRD patients receiving golimumab treatment. As TNFi treatments are increasingly used in clinical practice, the amount of real-world data will continue to grow and provide valuable information regarding relevant aspects of routine care.

While information regarding statistical predictors of persistence was extracted and summarised as part of the current review, underlying reasons for treatment switch or discontinuation as noted by a healthcare professional were not. Hence, it would be of interest to investigate reasons for the observed differences in persistence as part of future studies. Adverse effects, treatment efficacy and dose, out-of-pocket payments, patient characteristics (including, but not restricted to, the presence or absence of comorbidities) and patient preference with regard to eg, mode of administration have all been shown to impact the likelihood of treatment switch and/or discontinuation. ${ }^{56-58}$ Similarly, varying dosing intervals among subcutaneously administered TNFi treatments are relevant to consider. In the context of this review, golimumab is injected once monthly; adalimumab, every 2 weeks; certolizumab pegol, every 2-4weeks; and etanercept, once or twice weekly. As patients have been shown to prefer less frequent injections, ${ }^{56}{ }^{59}$ this difference might be expected to have an impact on patient preference and hence, persistence with treatment. The interaction between patient characteristics and reasons for differences in persistence would similarly be of interest to investigate, as it may yield valuable information regarding potential variations in the probability of remaining persistent with treatment between subgroups of patients. This type of information would be useful to healthcare 
practitioners, as it might be an initial step towards using different preventative measures directed at different patient groups for increasing treatment persistence.

\section{CONCLUSIONS}

In conclusion, the data from the studies included in this SLR indicate that in European routine clinical practice, golimumab has a high persistence at 12 and 24 months across different IMRD indications regardless of the treatment line.

\section{Author affiliations}

${ }^{1}$ Real World Evidence Strategy \& Analytics, ICON Clinical Research, Stockholm, Sweden

${ }^{2}$ Real World Evidence Strategy \& Analytics, ICON Clinical Research, Boston, Massachusetts, USA

${ }^{3}$ Karolinska Institutet, Stockholm, Sweden

${ }^{4}$ Medical Affairs Immunology, MSD Switzerland, Lucerne, Switzerland

${ }^{5}$ Center for Observational and Real-world Evidence (CORE), Merck \& Co., Inc.,

Kenilworth, New Jersey, USA

${ }^{6}$ Medical Affairs Rheumatology, MSD Italy, Rome, Italy

${ }^{7}$ Center for Observational and Real-world Evidence (CORE), MSD Sweden,

Stockholm, Sweden

Acknowledgements The authors would like to acknowledge the support of Axel Svedbom and Johan Dalén, who participated in initiating the study; in addition, the authors would also like to thank Birgitta Bayerl, who participated in performing the initial literature search.

Contributors CMB and NJ conceptualised the study. NJ developed the SLR protocol. MD performed the literature search. KL and MD performed article screening, data extraction, data analysis and drafted the manuscript. KL, MD, NJ, FC, SK, MG, SS, CMB and AK critically reviewed and substantially contributed to the final version of the manuscript.

Funding This work was funded by the Merck Sharp \& Dohme Corp., a subsidiary of Merck \& Co., Inc., Kenilworth, New Jersey, USA.

Competing interests KL, MD and NJ are employees of ICON PLC. FC, MG, SS and AK are employees of Merck Sharp \& Dohme, Lucerne, Switzerland. SK and CMB are employees of Merck Sharp \& Dohme Corp., a subsidiary of Merck \& Co., Inc., Kenilworth, New Jersey, USA.

Patient consent for publication Not required.

Provenance and peer review Not commissioned; externally peer reviewed.

Data sharing statement The study protocol is available upon request; please contact corresponding author.

Open access This is an open access article distributed in accordance with the Creative Commons Attribution Non Commercial (CC BY-NC 4.0) license, which permits others to distribute, remix, adapt, build upon this work non-commercially, and license their derivative works on different terms, provided the original work is properly cited, appropriate credit is given, any changes made indicated, and the use is non-commercial. See: http://creativecommons.org/licenses/by-nc/4.0/.

\section{REFERENCES}

1. Cantini F, Nannini C, Niccoli L. Bioboosters in the treatment of rheumatic diseases: a comprehensive review of currently available biologics in patients with rheumatoid arthritis, ankylosing spondylitis and psoriatic arthritis. Open Access Rheumatol 2009;1:163-78.

2. Zidi I, Bouaziz A, Mnif W, et al. Golimumab therapy of rheumatoid arthritis: an overview. Scand J Immunol 2010;72:75-85.

3. Gossec L, Smolen JS, Ramiro S, et al. European League Against Rheumatism (EULAR) recommendations for the management of psoriatic arthritis with pharmacological therapies: 2015 update. Ann Rheum Dis 2016;75:499-510.

4. Smolen JS, Landewé R, Bijlsma J, et al. EULAR recommendations for the management of rheumatoid arthritis with synthetic and biological disease-modifying antirheumatic drugs: 2016 update. Ann Rheum Dis 2017;76:960-77.

5. van der Heijde D, Ramiro S, Landewé R, et al. 2016 update of the ASAS-EULAR management recommendations for axial spondyloarthritis. Ann Rheum Dis 2017;76:978-91.

6. Simponi: EPAR - Product Information. Summary of product characteristics, 2018.

7. Keystone EC, Genovese MC, Klareskog L, et al. Golimumab, a human antibody to tumour necrosis factor \{alpha\} given by monthly subcutaneous injections, in active rheumatoid arthritis despite methotrexate therapy: the GO-FORWARD Study. Ann Rheum Dis 2009;68:789-96.

8. Inman RD, Davis JC, Heijde D, et al. Efficacy and safety of golimumab in patients with ankylosing spondylitis: results of a randomized, double-blind, placebo-controlled, phase III trial. Arthritis Rheum 2008;58:3402-12.

9. Kavanaugh A, van der Heijde D, Mclnnes IB, et al. Golimumab in psoriatic arthritis: one-year clinical efficacy, radiographic, and safety results from a phase III, randomized, placebo-controlled trial. Arthritis Rheum 2012;64:2504-17.

10. Sieper J, van der Heijde D, Dougados M, et al. A randomized, double-blind, placebo-controlled, sixteen-week study of subcutaneous golimumab in patients with active nonradiographic axial spondyloarthritis. Arthritis Rheumatol 2015;67:2702-12.

11. Deodhar A, Braun J, Inman RD, et al. Golimumab administered subcutaneously every 4 weeks in ankylosing spondylitis: 5-year results of the GO-RAISE study. Ann Rheum Dis 2015;74:757-61.

12. Kavanaugh A, Mclnnes IB, Mease P, et al. Clinical efficacy, radiographic and safety findings through 5 years of subcutaneous golimumab treatment in patients with active psoriatic arthritis: results from a long-term extension of a randomised, placebocontrolled trial (the GO-REVEAL study). Ann Rheum Dis 2014;73:1689-94.

13. Keystone EC, Genovese MC, Hall S, et al. Safety and Efficacy of Subcutaneous Golimumab in Patients with Active Rheumatoid Arthritis despite Methotrexate Therapy: Final 5-year Results of the GO-FORWARD Trial. J Rheumatol 2016;43:298-306.

14. Emery P, Fleischmann RM, Strusberg I, et al. Efficacy and Safety of Subcutaneous Golimumab in Methotrexate-Naive Patients With Rheumatoid Arthritis: Five-Year Results of a Randomized Clinical Trial. Arthritis Care Res 2016;68:744-52.

15. Smolen JS, Kay J, Doyle MK, et al. Golimumab in patients with active rheumatoid arthritis after treatment with tumour necrosis factor alpha inhibitors (GO-AFTER study): a multicentre, randomised, doubleblind, placebo-controlled, phase III trial. Lancet 2009;374:210-21.

16. Fries JF. Effectiveness and toxicity considerations in outcome directed therapy in rheumatoid arthritis. $J$ Rheumatol Suppl 1996;44:102-6.

17. Aletaha D, Smolen JS. Effectiveness profiles and dose dependent retention of traditional disease modifying antirheumatic drugs for rheumatoid arthritis. An observational study. $J$ Rheumatol 2002;29:1631-8.

18. Pincus T, Marcum SB, Callahan LF, et al. Longterm drug therapy for rheumatoid arthritis in seven rheumatology private practices: I. Nonsteroidal antiinflammatory drugs. J Rheumatol 1992;19:1874-84.

19. Cramer JA, Roy A, Burrell A, et al. Medication compliance and persistence: terminology and definitions. Value Health 2008;11:44-7.

20. Navarro-Millán I, Sattui SE, Curtis JR. Systematic review of tumor necrosis factor inhibitor discontinuation studies in rheumatoid arthritis. Clin Ther 2013;35:1850-61.

21. Arora A, Mahajan A, Spurden D, et al. Long-Term Drug Survival of TNF Inhibitor Therapy in RA Patients: A Systematic Review of European National Drug Registers. Int J Rheumatol 2013;2013:1-9.

22. Souto A, Maneiro JR, Gómez-Reino JJ. Rate of discontinuation and drug survival of biologic therapies in rheumatoid arthritis: a systematic review and meta-analysis of drug registries and health care databases. Rheumatology 2016;55:523-34.

23. Biggioggero M, Favalli EG. Ten-year drug survival of anti-TNF agents in the treatment of inflammatory arthritides. Drug Dev Res 2014;75(Suppl 1):S38-41.

24. Svedbom A, Storck C, Kachroo S, et al. Persistence with golimumab in immune-mediated rheumatic diseases: a systematic review of realworld evidence in rheumatoid arthritis, axial spondyloarthritis, and psoriatic arthritis. Patient Prefer Adherence 2017;11:719-29.

25. Moher D, Liberati A, Tetzlaff J, et al. Preferred reporting items for systematic reviews and meta-analyses: the PRISMA statement. PLOS Med 2009;6:e1000097.

26. Wells G, Shea B, O'Connell D. New Castle-Ottawa Quality Assessment Scale-Cohort Studies. 2012 http://www.ohri.ca/ programs/clinical_epidemiology/oxford.asp (15 Jun 2012). 
27. Aaltonen K, Heinonen A, Joensuu J, et al. Effectiveness and drug survival of TNF-inhibitors in the treatment of psoriatic arthritis: A prospective cohort study. Semin Arthritis Rheum 2017;46:732-9.

28. Aaltonen KJ, Joensuu JT, Pirilä L, et al. Drug survival on tumour necrosis factor inhibitors in patients with rheumatoid arthritis in Finland. Scand J Rheumatol 2017;46:359-63.

29. Dalén J, Svedbom A, Black CM, et al. Second-line treatment persistence and costs among patients with immune-mediated rheumatic diseases treated with subcutaneous TNF-alpha inhibitors. Rheumatol Int 2017;37:2049-58.

30. Dalén J, Svedbom A, Black CM, et al. Treatment persistence among patients with immune-mediated rheumatic disease newly treated with subcutaneous TNF-alpha inhibitors and costs associated with non-persistence. Rheumatol Int 2016;36:987-95.

31. Favalli EG, Sinigaglia $L$, Becciolini $A$, et al. Two-year persistence of golimumab as second-line biologic agent in rheumatoid arthritis as compared to other subcutaneous tumor necrosis factor inhibitors: real-life data from the LORHEN registry. Int $J$ Rheum Dis 2018;21:422-30.

32. lannone F, Santo L, Anelli MG, et al. Golimumab in real-life settings: 2 Years drug survival and predictors of clinical outcomes in rheumatoid arthritis, spondyloarthritis, and psoriatic arthritis. Semin Arthritis Rheum 2017:47:108-14

33. Manara M, Caporali R, Favalli EG, et al. Two-year retention rate of golimumab in rheumatoid arthritis, psoriatic arthritis and ankylosing spondylitis: data from the LORHEN registry. Clin Exp Rheumatol 2017;35:804-9.

34. Mourão AF, Ribeiro C, Borges J, et al. Real-life effectiveness of Golimumab in biologic-naïve patients with rheumatoid arthritis - data from the Rheumatic Diseases Portuguese Register (Reuma.pt). Acta Reumatol Port 2017;42:141-9.

35. Thomas K, Flouri I, Repa A, et al. High 3-year golimumab survival in patients with rheumatoid arthritis, ankylosing spondylitis and psoriatic arthritis: real world data from 328 patients. Clin Exp Rheumatol 2018;36:254-62.

36. Belhassen M, Hudry C, Woronoff M, et al. Treatment Persistence with Golimumab in France. Value in Health 2016;19:A592.

37. Belhassen M. Treatment persistence with subcutaneous TNF-alpha inhibitors in France. Arthritis and Rheumatology 2016:2926-7.

38. Fautrel B. Determinants of 12-months persistence in ankylosing spondylitis patients initiating subcutaneous TNF-alpha inhibitors: EULAR, 2017.

39. Fautrel B. Determinants of 12-months persistence in psoriatic arthritis patients initiating subcutaneous TNF-alpha inhibitors: EULAR, 2017.

40. Fautrel B. Determinants of 12-months persistence in rheumatoid arthritis patients initiating subcutaneous TNF-alpha inhibitors: EULAR, 2017.

41. Favalli EG, Caporali R, Monti S, et al. FRI0174 Two-Year Retention Rate of Subcutaneous Anti-Tumor Necrosis Factor Agents for Rheumatoid Arthritis: A Retrospective Analysis of The Lorhen Registry. Ann Rheum Dis 2016;75:492.2-492.

42. Flipo RM. The real-life use of golimumab in patients with immunemediated rheumatic diseases: one year results of the go-practice study. Annals of the Rheumatic Diseases 2017:1299.

43. García-Porrúa C. Drug survival on first TNF inhibitors in patients with psoriatic arthritis: Comparison across etanercept, adalimumab, golimumab and infliximab: EULAR, 2018.
44. González Fernández CM, et al. Persistence on golimumab as second line biological therapy in patients with spondyloarthritis (axial spondyloarthritis and psoriatic arthritis). GO-BEYOND, a retrospective study, in EULAR 2018.

45. Hernández MV. Factors associated to persistence on golimumab in patients with inflammatory arthritis of the BIOBADASER registry: EULAR, 2018.

46. lannone $\mathrm{F}$, et al. Clinical outcomes of treatment with golimumab in seropositive and seronegative rheumatoid arthritis patients in real-life settings. Data from Italian register GISEA., in EULAR 2018.

47. Klaudius I. Golimumab improves work productivity and activity impairment in patients with rheumatoid arthritis (RA), ankylosing spondylitis (AS) and psoriatic arthritis (PSA): 1-year results from a non-interventional trial in Germany: EULAR, 2018.

48. Michelsen B, Sexton J, Kvien TK. Does biological diseasemodifying anti-rheumatic drug naïve versus non-naïve patients with inflammatory joint diseases have similar golimumab drug survival and efficacy? Data from the prospective observational NOR-DMARD study. EULAR 2018.

49. Santo L. Effectiveness of golimumab in TNF-inhibitor inadequate responder patients with spondyloarthritis and psoriatic arthritis. EULAR 2017

50. Scrivo R. An Italian observational prospective study on predictors of clinical response to golimumab at 6 months in patients with active psoriatic arthritis. EULAR 2018.

51. Serrano-Benavente B. Golimumab retention rate in patients with spondyloarthritis. Differences between ankylosing spondylitis and non-radiographic axial spondyloarthritis. EULAR 2018.

52. Serrano-Benavente $B$. Long-term golimumab retention rate in patients with psoriatic arthritis. Is concomitant DMARD important? EULAR 2018

53. Svedborn A, Dalen J, Lyu R, et al. THU0370 Treatment Persistence Among Patients with Rheumatoid Disease (RD) Newly Treated with Subcutaneous TNF-Alpha Blockers. Ann Rheum Dis 2015;74:330.1-330.

54. Gu T, Mutebi A, Stolshek BS, et al. Cost of biologic treatment persistence or switching in rheumatoid arthritis. Am J Manag Care 2018;24(8 Spec No.):SP338-45.

55. Giacomelli R, Ruscitti P, Bombardieri S, et al. What could we learn from the sub-analysis of a single nation cohort in a worldwide study? Lessons from the results observed in the Italian cohort of the GOMORE trial. Clin Exp Rheumatol 2017;35:623-9.

56. Giacomelli R, Gorla R, Trotta F, et al. Quality of life and unmet needs in patients with inflammatory arthropathies: results from the multicentre, observational RAPSODIA study. Rheumatology 2015;54:792-7.

57. Murage MJ, Tongbram V, Feldman SR, et al. Medication adherence and persistence in patients with rheumatoid arthritis, psoriasis, and psoriatic arthritis: a systematic literature review. Patient Prefer Adherence 2018;12:1483-503.

58. Schulze-Koops H, Giacomelli R, Samborski W, et al. Factors influencing the patient evaluation of injection experience with the SmartJect autoinjector in rheumatoid arthritis. Clin Exp Rheumatol 2015;33:201-8

59. Bhoi P, Bessette L, Bell MJ, et al. Adherence and dosing interval of subcutaneous antitumour necrosis factor biologics among patients with inflammatory arthritis: analysis from a Canadian administrative database. BMJ Open 2017;7:e015872. 